\title{
Efficient synthesis of 2,5-dicarbonyl derivatives of 3,4- ethylenedithiothiophene (EDTT) via addition-elimination reaction
}

\author{
Mustafa A-jabbar Al-jumaili ${ }^{\mathrm{a}, \mathrm{b}}$, Simon Woodward ${ }^{\mathrm{a}, \text {, }}$ \\ ${ }^{a}$ GSK Carbon Neutral Laboratories for Sustainable Chemistry, University of Nottingham, Jubilee \\ Campus, Nottingham NG7 2TU, United Kingdom \\ ${ }^{\mathrm{b}}$ Ministry of Planning in Iraq, Central Organization for Standardization and Quality Control, \\ Baghdad, Al-Jadriya, Iraq
}

\begin{abstract}
Derivatives of 3,4-ethylenedithiothiophene (EDTT) are reported starting from tetrabromothiophene. Selective 2,5-dilithiation followed by reaction with a range of aldehydes gives diols as mixtures of diastereomers. Only the 2 and 5 positions in thiophene react leaving the 3,4-bromides for further elaboration. The diols are oxidised to their corresponding diketones using activated $\mathrm{MnO}_{2}$. Reaction with 1,2-ethanedithiol, by addition-elimination, provides access to novel monomers for the preparation of conjugated copolymers of 3,4-ethylenedithiothiophene (EDTT). A range of these monomers can be attained by applying the synthesis of a series of ketones applicable to further synthesis of $\pi$-extended thiophene-based organic semiconductors. Finally, this new route was compared to 3,4ethylenedioxythiophene (EDOT) dialdehyde derivatives synthesised by an alternative to literature chemistry.
\end{abstract}

Keywords: Addition-elimination reaction; 3,4-Ethylenedithiothiophene; 1,2-Ethanedithiol; Organic semiconductors; EDTT

\section{Introduction}

From their initial mainstream uptake in the early 1980s oligothiophenes have risen to become one of the most privileged structures in the organic semi-conducting materials area. ${ }^{1,2}$ By appropriate choice of thiophene substituents and microstructure (e.g. degree of oligomerisation, connectivity, use of additives, etc.) the resultant materials' (opto)electrical properties can be modulated with a high degree of precision. This ability to tune thiophene based materials for a specific application has led to their widespread use in organic photovoltaics (OPV), ${ }^{3}$ organic field effect transistors (OFET),${ }^{4}$ sensors ${ }^{5}$ and particularly in electrochromic/electroluminescent (OLED) ${ }^{6}$ applications.

Such 'modular-materials' approaches have created an insatiable appetite for new thiophene sub-units from materials chemists for their inclusion into oligo(poly)thiophene structures as evidenced by the thousands of papers that have appeared in this area in the last 10 years. In particular, derivatives of 3,4ethylenedioxythiophene (EDOT) 1 have proved popular targets as this monomer is easily polymerised to PEDOT-based polymers $\mathbf{3}^{7}$ whose formulation with polymer supported sulfonates (PEDOT-PSS) now forms the backbone of many transparent electrode technologies. Recently, new thermoelectric (TE) applications for PEDOT-based materials have emerged, ${ }^{8}$ potentially allowing direct conversion of waste heat into electrical power. For optimal TE devices the use of bulk, rather than thin film, configurations would be preferred (to maximise power-from-heat recovery). Although the low band gap of PEDOTPSS $\left(1.6 \mathrm{eV}\right.$ ) favours high electrical conductivity ${ }^{9}$ (vital for TE applications) the physical properties of current PEDOT-PSS co-polymers (solubility, $\mathrm{mp}$ ) are less favourable for fabrication of bulk $(\mathrm{mm})$ rather than thin film $(\sim 100 \mathrm{~nm})$ devices. We considered the possibility that derivatives of the sulfur analogue of EDOT, that is EDTT 2, might prove of utility in seeking more processible entities. Both EDTT itself and PEDTT are known versatile components in organic materials chemistry. ${ }^{10}$ Although the band gap of PEDTT 4 is greater $(2.2 \mathrm{eV})$ than PEDOT, building in functionality to allow the creation 
of vinyligous linkages at the 2,5-postitions of EDTT 2 should allow band gap reduction, as similar synergies have been noted in related structures. ${ }^{11}$ The positioning of suitable groups to promote solubility and processability of bulk samples is also desirable.

Consequently, two main points are investigated in this paper. Firstly, the synthesis of new polymer precursors based on EDTT 2 that might have lower band gap potentials (essential for organic materials applications) even if these are based on PEDTT-like structures. Secondly, the potential structure of the new polymers should allow for the improved solubility characteristics required for many applications. Two general strategies are employed here in to target monomers for new polymers having small HOMO-LUMO energy differences and good solubility in the different solvents. The first involves increasing the conjugation of polymers by inserting an alkenyl unit $(\mathrm{C}=\mathrm{C})$ into the structure of the polymers, to attain a lower band gap. The second strategy involves strategic placement of alkyl groups whereby the side chain will increase the polymer solubility and thus improve its processability (see Scheme 1).
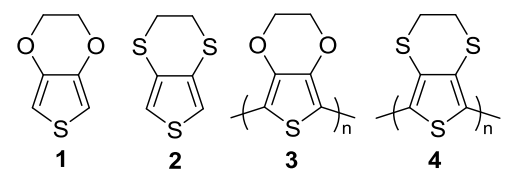

Scheme 1. The structures of EDOT 1 and EDTT 2 and their derived polymers PEDOT 3 and PEDTT 4.

\section{Results and discussion}

\subsection{Synthetic routes to derivatives of EDTT}

Our initial study focused on methods to quickly and reliably attain 2,5-derivatives of 3,4ethylenedithiothiophene (EDTT) $\mathbf{2}$ starting from the readily available crystalline tetrabromothiophene 5 (Scheme 2).
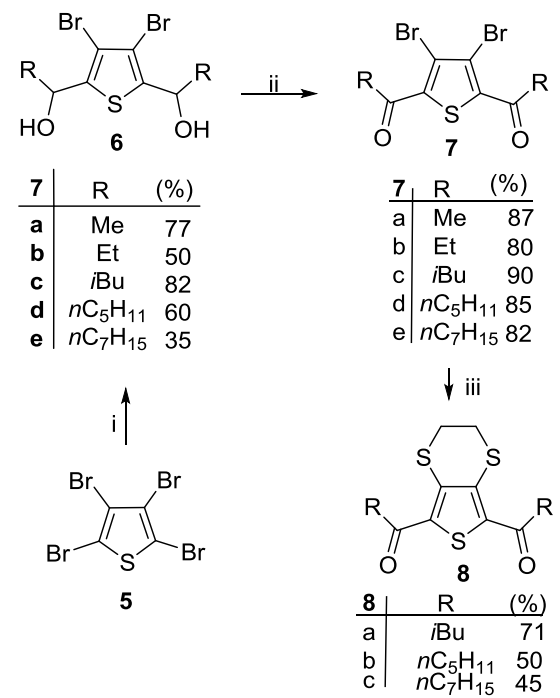

Scheme 2. Synthetic route to ketothiophenes. Reagents and conditions: (i) THF/nBuLi, RCHO, -78 to $-20{ }^{\circ} \mathrm{C}$, $3 \mathrm{~h}$; (ii), $\mathrm{MnO}_{2}$ (10 equiv.), $\mathrm{CH}_{2} \mathrm{Cl}_{2}, 40{ }^{\circ} \mathrm{C}, 16 \mathrm{~h}$; (iii) 1,2-ethanedithiol (2.2 equiv.), $\mathrm{DMF}, \mathrm{Na}_{2} \mathrm{CO}_{3}, 16$ h, r.t.

Tetrabromothiophene $\mathbf{5}$ is easily prepared on large scales by bromination of thiophene in $73 \%$ yield, the $\mathrm{HBr}$ by-product is conveniently quenched by collecting the off-gas into a suitable water trap. The activated 2,5 positions of $\mathbf{5}$ are cleanly exchanged with $n \mathrm{BuLi}$ at $-78^{\circ} \mathrm{C}$. While rearrangements (the so called "halogen dance" ${ }^{12}$ ) can take place at higher temperatures, reactive electrophiles are expected to intercept the dilithiated intermediate cleanly. Relatively few investigations have employed this strategy 
but examples with: chlorotrimethylsilane, ${ }^{13}$ a limited number of aldehydes ${ }^{14}$ and acyl chlorides ${ }^{15}$ are known. However, the reported yields can be poor in such procedures.

From a practical perspective, addition of the aldehyde at $-78^{\circ} \mathrm{C}$ followed by warming to $-20^{\circ} \mathrm{C}$ affords the maximum yield of 6a-e (40-82\%) by minimising organolithium rearrangement. Compounds $\mathbf{6 a - e}$ are attained as a diastereomeric mixture but the syn and anti diastereomers could be separated by careful column chromatography. Each of the diastereomeric compounds 6a-e could be converted into the corresponding ketones 7a-e using activated $\mathrm{MnO}_{2}$, in refluxing dichloromethane. Simple overnight reaction is required to attain complete conversion. Ketones $\mathbf{8}$ are easily isolated by column chromatography as they appear as bright yellow bands. The resulting micro-crystalline yellow solids show spectra in accord with the proposed formulation. The presence of two keto functions allows potentially many condensation polymers to be accessed related, in part, to EDTT 2 and PEDTT 4.

For comparison, the closest literature method for the synthesis of EDTT 2 we could identify is shown in Scheme $3 .{ }^{16}$ This approach requires two steps from 9 to add the bis-thioether in $86 \%$ overall yield. In our approach (Scheme 2), compounds 8a-c require only one step to install the same thioether. By the reaction of 1,2-ethanedithiol with compounds 7a-c (in DMF and over 2 days in the presence of $\mathrm{Na}_{2} \mathrm{CO}_{3}$ ) more modest yields of 71, 50 and $45 \%$ are attained for 8a-c respectively. For further comparison, the parent $\mathbf{2}$, and its S,O-analogue, can be also prepared from $\mathbf{9}$ by the chemistry of Roncali. ${ }^{17}$

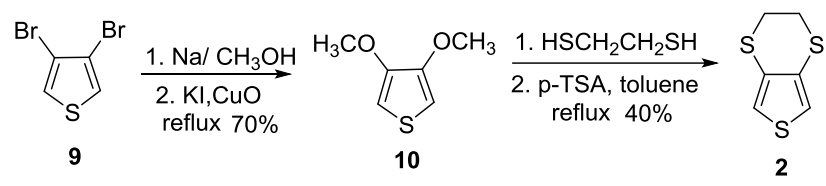

Scheme 3. One comparitive route for EDTT 2. $\frac{16}{}$.

\subsection{Synthetic routes to derivatives of EDOT}

In the last few years 3,4-ethylenedioxythiophene (EDOT) $\mathbf{1}$ and its derivatives have become very important commercial compounds for the preparation of new organic materials. ${ }^{7,18}$ However, most commercial routes to EDOT use $\mathrm{S}\left(\mathrm{CH}_{2} \mathrm{CO}_{2} \mathrm{Me}\right)_{2}$ and rely on late stage (wasteful) decarboxylation of the ester units. We wondered if a nitrile group was used instead if this could provide access to 2,5disubstituted aldehydes closely related to our diketo-EDTT species $\mathbf{8}$. This new route to derivatives of EDOT has been investigated as shown in Scheme 4.
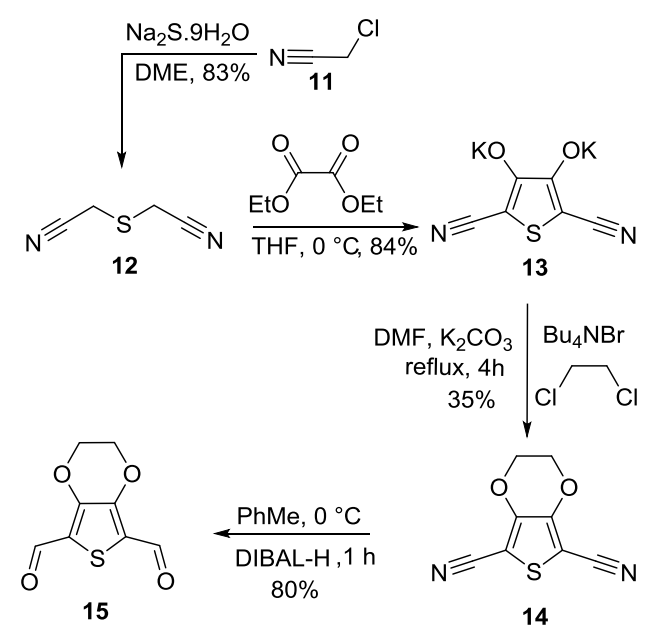

Scheme 4. Synthetic route for the derivatives of EDOT 
The intermediate $\mathbf{1 3}$ is prepared in two steps, the first uses the ultra-cheap starting material $\mathrm{Na}_{2} \mathrm{~S} \cdot 9 \mathrm{H} 2 \mathrm{O}$ for alkylation with chloroacetonitrile $\mathbf{1 1}$ in DME to yield compound $\mathbf{1 2}$ near quantitatively and in excellent purity which allows its use without purification. Subsequent reaction of $\mathbf{1 2}$ and diethyl oxalate prepares the potassium salt $\mathbf{1 3}$, which is also used directly without further purification, to access the dinitrile $\mathbf{1 4}$ in modest yield. However, $\mathbf{1 4}$ is easily isolated analytically pure after a simple filtration through a silica plug. Finally, $\mathbf{1 4}$ was converted to the corresponding aldehyde $\mathbf{1 5}$ by its treatment with DIBAL-H at $0{ }^{\circ} \mathrm{C}$ in anhydrous toluene. Compound $\mathbf{1 5}$ is already recognised as an important intermediate in the synthesis of thiophene-based semiconductor polymers. ${ }^{19,20}$

\section{Conclusions}

We have successfully found a new route to homologs of 3,4-ethylenedithiothiophene (EDTT) 2 having ketone functionalities at the 2,5-positions. From tetrabromothiophene 5, monomers 8a-c were obtained in three steps. In addition, the monomers 8a-c are attractive species for the future synthesis of many thiophene-based semiconductor polymers. Additionally, we have reported a new route for the synthesis of the 2,5-diformyl derivative of 3,4-ethylenedioxythiophene (EDOT), the key intermediate $\mathbf{1 4}$ is obtained using ultra-cheap $\mathrm{Na}_{2} \mathrm{~S} \cdot 9 \mathrm{H}_{2} \mathrm{O}$ in just three steps. Finally, nitrile to formyl conversion proceeds cleanly in near quantitative yield.

\section{Experimental}

General. All reagents and solvents were purchased from Sigma-Aldrich and were used as supplied unless otherwise stated. Tetrahydrofuran was freshly distilled under argon from sodium/benzophenone. Aldehydes were distilled before use. Evaporation of the solvents was completed by rotary evaporation under reduced pressure. All temperatures refer to those of the cooling and heating baths used. Cooling of the reactions was achieved using a Haake DC50-K75 refrigerated circulator. Thin layer chromatography was performed on foil-backed plates plated with Merck Silica gel $60 \mathrm{~F}_{254}$. The plates were visualised using ultraviolet light and basic aqueous $\mathrm{KMnO}_{4}$. Liquid chromatography was carried out using flash column with specific solvent systems. All proton spectra were referenced to $\mathrm{CDCl}_{3}(\delta=7.27 \mathrm{ppm})$ or acetone- $\mathrm{d}_{6}(\delta=2.05 \mathrm{ppm})$ as an internal standard. All ${ }^{13} \mathrm{C}$ NMR samples were proton-decoupled and referenced to $\mathrm{CDCl}_{3}(\delta=77.0 \mathrm{ppm})$, acetone- $\mathrm{d}_{6}(\delta=29.9 \mathrm{ppm})$ or DMSO$d_{6}(\delta=39.5 \mathrm{ppm})$. Coupling constants $(J)$ are quoted in Hertz. Infrared spectra were recorded with a Varian FTS-7000 FT-IR spectrometer using (ATR) operating at room temperature. Mass spectrometry was performed using a Bruker MicroTOF or VG Micromass AutoSpec spectrometers using electrospray (ESI), electron impact (EI) ionization modes; theoretical HRMS molecular weights were determined by these instrument's software. The starting materials tetrabromothiophene 5, 2,2'thiodiacetonitrile 12 and activated $\mathrm{MnO}_{2}$ were prepared by modifications of literature procedures but are described here for clarity. Activated $\mathrm{MnO}_{2}$ was prepared by a literature method. ${ }^{21}$

\subsection{Tetrabromothiophene (5)}

Compound $\mathbf{5}$ was prepared by modification of a literature methods. ${ }^{22}$ Thiophene $(10.6 \mathrm{~g}, 0.127 \mathrm{~mol})$ dissolved in chloroform $(8 \mathrm{~mL})$ was treated with a solution of bromine $(24 \mathrm{~mL}, 0.46 \mathrm{~mol})$ in chloroform $(8 \mathrm{~mL})$ that was added dropwise over $50 \mathrm{~min}$ at $0{ }^{\circ} \mathrm{C}$. The resulting dark mixture was stirred overnight at room temperature, then additional bromine $(9.0 \mathrm{~mL}, 0.17 \mathrm{~mol})$ was then added and the mixture refluxed $\left(70{ }^{\circ} \mathrm{C}, 57 \mathrm{~h}\right)$. After the reaction had cooled to room temperature, saturated potassium hydroxide solution $(100 \mathrm{~mL})$ was added over a 10 -min period and the mixture refluxed again $\left(105^{\circ} \mathrm{C}\right.$, $18 \mathrm{~h}$ ). The reaction was stopped and allowed to cool to room temperature. The 2,3,4,5tetrabromothiophene 5 was extracted with dichloromethane, and dried over $\mathrm{MgSO}_{4}$, and solvent removed. The crude compound was attained as analytically pure colourless crystalline needles $(39.6 \mathrm{~g}$, 99.0 mmol, $78 \%$ ); m.p. $114-115{ }^{\circ} \mathrm{C}$ (lit. $\left..^{22} 116-118{ }^{\circ} \mathrm{C}\right) ;{ }^{13} \mathrm{C} \mathrm{NMR}\left(100 \mathrm{MHz}, \mathrm{CDCl}_{3}\right) \delta_{\mathrm{C}} 117.1,110.4$; 
MS (EI): $m / z 400\left(\mathrm{M}^{+}\right)$, found $395.6467 \mathrm{C}_{4} \mathrm{SBr}_{4}$ requires $395.6448\left({ }^{79} \mathrm{Br}\right)$. These properties were in accord with the literature. ${ }^{22}$

\subsection{General preparation of the diols (6), representative procedure, 1,1'-(3,4-dibromothiophene- 2,5-diyl)bis(1-ethanol) (6a)}

To a solution of tetrabromothiophene $\mathbf{5}(1.10 \mathrm{~g}, 2.75 \mathrm{mmol})$ in dry THF $(9 \mathrm{~mL})$ under argon, $n \mathrm{BuLi}$ (3.77 $\mathrm{mL}, 1.5 \mathrm{M}$ in hexane, $5.66 \mathrm{mmol})$ was added dropwise at $-78^{\circ} \mathrm{C}$. Excess acetaldehyde $(0.5 \mathrm{~mL})$ was added at $-78^{\circ} \mathrm{C}$. The temperature of the mixture was allowed to warm slowly during $2 \mathrm{~h}$ from $(-78$ to $\left.-25{ }^{\circ} \mathrm{C}\right)$. The reaction was quenched with water $(8 \mathrm{~mL})$, extracted with EtOAc, dried $\left(\mathrm{MgSO}_{4}\right)$ and evaporated to a brown oil. Flash column chromatography (7:3 pentane:EtOAc) yielded a colourless solid $(0.696 \mathrm{~g}, 77 \%)$ as a mixture of diastereomers (1:1); $\mathrm{R} f$ values $0.37,0.44$ (7:3 pentane:EtOAc); the m.p. for the mixture of diastereomers is $96-110{ }^{\circ} \mathrm{C} ;{ }^{1} \mathrm{H} \mathrm{NMR}\left(400 \mathrm{MHz}\right.$, acetone- $\left.\mathrm{d}_{6}\right) \delta_{\mathrm{H}} 5.16-5.10(\mathrm{~m}$, $\left.2 \mathrm{H}, \mathrm{CH}_{3} \mathrm{CH}\right), 4.89-4.88(\mathrm{~m}, 2 \mathrm{H}, \mathrm{OH}), 1.46-1.44\left(\mathrm{~m}, 6 \mathrm{H}, \mathrm{CHCH}_{3}\right) ;{ }^{13} \mathrm{C}$ NMR $(100 \mathrm{MHz}$, acetoned 6 ) $\delta_{\mathrm{C}} 146.0,107.9,66.9,24.8$; IR(ATR) $\left(\mathrm{cm}^{-1}\right) v_{\max } 3285,2974,2925,1436,1397,1368,1329,1273$, 1177, 1088, 1061, 983, 885, 861, 764; MS (ESI): found 329.8747, calcd. for $\mathrm{C}_{8} \mathrm{H}_{10} \mathrm{Br}_{2} \mathrm{O}_{2} \mathrm{~S} 329.8748$ $\left({ }^{79} \mathrm{Br}\right)$.

\subsection{1,1'-(3,4-dibromothiophene-2,5-diyl)bis(1-propanol) (6b)}

Using tetrabromothiophene $\mathbf{5}(0.50 \mathrm{~g}, 1.25 \mathrm{mmol})$ in dry THF $(3.2 \mathrm{~mL})$ under argon, $n \mathrm{BuLi}(1.8 \mathrm{~mL}$, $1.5 \mathrm{M}$ in hexane, $2.75 \mathrm{mmol})$ was added dropwise at $-78{ }^{\circ} \mathrm{C}$. Then excess propanal $(0.27 \mathrm{~mL}$, $3.75 \mathrm{mmol}$ ) was added at $-78{ }^{\circ} \mathrm{C}$. The temperature of the mixture was warmed slowly during $3 \mathrm{~h}$ from $\left(-78\right.$ to $\left.-65^{\circ} \mathrm{C}\right)$. The reaction was quenched with water $(5 \mathrm{~mL})$ extracted with EtOAc, dried $\left(\mathrm{MgSO}_{4}\right)$, and evaporated to a brown crude product purified by flash column chromatography (8.5:1.5 light petroleum:EtOAc) yielding a yellow oil $(0.215 \mathrm{~g}, 50 \%)$ as mixture of diastereomers $(2: 1)$; $\mathrm{R} f$ values 0.10, 0.07 (17:3 light petroleum:EtOAc); ${ }^{1} \mathrm{H}$ NMR (400 MHz, $\left.\mathrm{CDCl}_{3}\right) \delta_{\mathrm{H}} 4.89(\mathrm{~m}, 2 \mathrm{H}, \mathrm{CH}), 1.73-1.65$ $\left(\mathrm{m}, 4 \mathrm{H}, \mathrm{CH}_{2}\right), 1.00(\mathrm{t}, J=7.3 \mathrm{~Hz}, 6 \mathrm{H})$, the $\mathrm{OH}$ signals were not observed due to exchange; ${ }^{13} \mathrm{C}$ NMR $\left(100 \mathrm{MHz}, \mathrm{CDCl}_{3}\right) \delta_{\mathrm{C}} 143.9,108.7,71.6,32.0,10.4$; IR(ATR) $\left(\mathrm{cm}^{-1}\right) v_{\max } 3313,2964,2930,2874$, 1458, 1378, 1319, 1319, 1171, 1089, 1041, 971, 868; MS (ESI): found 355.9092, calcd. for $\mathrm{C}_{10} \mathrm{H}_{14} \mathrm{Br}_{2} \mathrm{O}_{2} \mathrm{~S}\left({ }^{79} \mathrm{Br}\right) 355.9075$.

\subsection{1,1'-(3,4-dibromothiophene-2,5-diyl)bis(3-methylbutanol) (6c)}

Preparation from tetrabromothiophene $5(2.00 \mathrm{~g}, 5.00 \mathrm{mmol}, n \mathrm{BuLi}(6.9 \mathrm{~mL}, 1.6 \mathrm{M}$ in hexane, $11.0 \mathrm{mmol})$ and methylbutyraldehyde $(1.29 \mathrm{~g}, 15.0 \mathrm{mmol})$. The brown crude product was purified by flash column chromatography (85:15 light petroleum:EtOAc) to yield a pale yellow solid as a (2:1) mixture of diastereomers (1.70 g, 82\%); Rf values 0.31, 0.15 (85:15 light petroleum:EtOAc); m.p. 112 $113{ }^{\circ} \mathrm{C} ;{ }^{1} \mathrm{H} \mathrm{NMR}\left(400 \mathrm{MHz}, \mathrm{CDCl}_{3}\right) \delta_{\mathrm{H}} 5.14-5.13(\mathrm{~m}, 2 \mathrm{H}, 2 \times \mathrm{CHOH}), 2.22(\mathrm{br}, 2 \mathrm{H}, 2 \times \mathrm{OH}), 1.82-$ $1.57\left(\mathrm{~m}, 6 \mathrm{H}, 2 \times \mathrm{CH}_{2}, 2 \times \mathrm{CH}\right) ; 1.04-0.96\left(\mathrm{~m}, 12 \mathrm{H}, 4 \times \mathrm{CH}_{3}\right) ;{ }^{13} \mathrm{C} \mathrm{NMR}\left(100 \mathrm{MHz}, \mathrm{CDCl}_{3}\right) \delta_{\mathrm{C}} 143.1$, 110.0, 68.8, 47.2, 24.9, 23.3, 22.1; IR(ATR) $\left(\mathrm{cm}^{-1}\right) v_{\max } 3220,2955,2923,2868,1743,1467,1384$, $1367,1236,1180,1108,1067,846,767,679,595$; MS (ESI): found 413.9688, calcd. for $\mathrm{C}_{14} \mathrm{H}_{22} \mathrm{Br}_{2} \mathrm{O}_{2} \mathrm{~S}$ $413.9687\left({ }^{79} \mathrm{Br}\right)$.

\subsection{1,1'-(3,4-dibromothiophene-2,5-diyl)bis(1-hexanol)(6d)}

Preparation from tetrabromothiophene $5(1.00 \mathrm{~g}, 2.50 \mathrm{mmol}, n \mathrm{BuLi}(3.7 \mathrm{~mL}, 1.5 \mathrm{M}$ in hexane, $5.50 \mathrm{mmol})$ and $n$-hexanal $(0.9 \mathrm{~mL}$, ca. $0.75 \mathrm{~g}, 7.5 \mathrm{mmol})$. The brown crude product was purified by flash column chromatography (85:15 light petroleum:EtOAc) to yield of pale yellow solid as (1:1) mixture of diastereomers $(0.80 \mathrm{~g}, 72 \%)$ as colourless solid; $\mathrm{R} f 0.66,0.37$ (6:1 pentane:EtOAc); m.p. 82-84 ${ }^{\circ} \mathrm{C} ;{ }^{1} \mathrm{H}$ NMR $\left(400 \mathrm{MHz}, \mathrm{CDCl}_{3}\right) \delta_{\mathrm{H}} 5.24(\mathrm{~s}, 2 \mathrm{H}, 2 \times \mathrm{OH}), 4.93(\mathrm{~m}, 2 \mathrm{H}, 2 \times \mathrm{CH}), 1.64-1.36(\mathrm{~m}$, $\left.16 \mathrm{H}, 2 \times \mathrm{CH}_{2} \mathrm{CH}_{2} \mathrm{CH}_{2} \mathrm{CH}_{2}\right), 0.92\left(\mathrm{t}, J=7.3 \mathrm{~Hz}, 6 \mathrm{H}, 2 \times \mathrm{CH}_{3}\right) ;{ }^{13} \mathrm{C} \mathrm{NMR}\left(100 \mathrm{MHz}, \mathrm{CDCl}_{3}\right) \delta_{\mathrm{C}} 144.2$, 108.0, 70.1, 38.7, 31.5, 25.8, 22.8, 14.2; IR(ATR) $\left(\mathrm{cm}^{-1}\right) v_{\max } 3296,2954,2921,2851,1642,1464,1367$, 
1316, 1248, 1182, 1164, 1058, 971, 927, 769; MS (ESI): found 442.0007, calcd. for $\mathrm{C}_{16} \mathrm{H}_{2} 6 \mathrm{Br}_{2} \mathrm{O}_{2} \mathrm{~S}\left({ }^{79} \mathrm{Br}\right)$ 442.0000 .

\subsection{1,1'-(3,4-dibromothiophene-2,5-diyl)bis(octan-1-ol) (6e)}

Preparation from tetrabromothiophene $5(3.00 \mathrm{~g}, 7.50 \mathrm{mmol}, n \mathrm{BuLi}(11.0 \mathrm{~mL}, 1.5 \mathrm{M}$ in hexane, $16.51 \mathrm{mmol})$ and $n$-octanal $(2.40 \mathrm{~g}, 18.76 \mathrm{mmol})$. The yellow crude product was purified by flash column chromatography (9:1 light petroleum:EtOAc) to yield a yellow oil as a (2:1) mixture of diastereomers (1.46 g, 40\%); $\mathrm{R} f$ values 0.30, 0.20 (9:1 light petroleum:EtOAc); ${ }^{1} \mathrm{H}$ NMR $(400 \mathrm{MHz}$, $\left.\mathrm{CDCl}_{3}\right) \delta_{\mathrm{H}} 5.06-5.02(\mathrm{~m}, \quad 2 \mathrm{H}, \quad 2 \times \mathrm{CH}), \quad 1.83-1.77 \quad\left(\mathrm{~m}, \quad 4 \mathrm{H}, \quad 2 \times \mathrm{CH}_{2}\right), \quad 1.31 \quad(\mathrm{~m}, \quad 20 \mathrm{H}$, $\left.2 \times \mathrm{CH}_{2} \mathrm{CH}_{2} \mathrm{CH}_{2} \mathrm{CH}_{2} \mathrm{CH}_{2}\right), 0.88\left(\mathrm{t}, J=6.9 \mathrm{~Hz}, 6 \mathrm{H}, 2 \times \mathrm{CH}_{3}\right)$ ), the $\mathrm{OH}$ signals $\sim \delta_{\mathrm{H}} 2.4$ were often not clearly observed due to exchange; ${ }^{13} \mathrm{C}$ NMR $\left(100 \mathrm{MHz}, \mathrm{CDCl}_{3}\right) \delta_{\mathrm{C}} 142.8,110.1,70.6,38.1,31.9,29.4$, 29.3, 25.7, 22.8, 14.2; IR(ATR) $\left(\mathrm{cm}^{-1}\right) v_{\max } 3331,2954,2922,2853,1463,1315,1168,1050,1019,907$, 765; MS (ESI): found 496.0619, calcd. for $\mathrm{C}_{20} \mathrm{H}_{34} \mathrm{Br}_{2} \mathrm{O}_{2} \mathrm{~S} 496.0640$.

\subsection{General procedure for oxidation of compounds (6a-e) to corresponding ketone}

Diols $6(1.41 \mathrm{mmol})$ were dissolved in dichloromethane $(20 \mathrm{~mL})$, and activated $\mathrm{MnO}_{2}{ }^{21}(10$ molar equivalents) added. The dark black suspension was stirred $(16 \mathrm{~h})$ at $40-45{ }^{\circ} \mathrm{C}$. Completion of the reaction was determined by TLC. After filtration through Celite, the reaction mixture was concentrated in vacuo to afford the corresponding ketone products as pale solids in about $90 \%$ yield. These products were $>98 \%$ pure by ${ }^{1} \mathrm{H}$ NMR spectroscopy and used without further purification.

\subsection{1,1'-(3,4-dibromothiophene-2,5-diyl)bis(ethan-1-one) (7a)}

Prepared from diol 6a (55 mg, $0.16 \mathrm{mmol}$ ) to obtain compound $7 \mathbf{a}$ as a colourless solid (47 $\mathrm{mg}, 87 \%$ ). Rf 0.84 (7:3 pentane/EtOAc), m.p. $168-170{ }^{\circ} \mathrm{C} \quad\left[\right.$ lit. $\left..20173-174{ }^{\circ} \mathrm{C}\right] ;{ }^{1} \mathrm{H} \quad \mathrm{NMR} \quad(400 \mathrm{MHz}$, $\left.\mathrm{CDCl}_{3}\right) \delta_{\mathrm{H}} 2.74\left(\mathrm{~s}, 6 \mathrm{H}, 2 \times \mathrm{CH}_{3}\right) ;{ }^{13} \mathrm{C} \mathrm{NMR}\left(100 \mathrm{MHz}, \mathrm{CDCl}_{3}\right) \delta_{\mathrm{C}} 189.7,142.7,119.4,29.9$; IR(ATR) $\left(\mathrm{cm}^{-1}\right) v_{\max } 2919,2852,1649,1474,1414,1353,1240,1044,1029,949,870,802,666 ;$ MS (ESI): found 325.8431, calcd. for $\mathrm{C}_{8} \mathrm{H}_{6} \mathrm{Br}_{2} \mathrm{O}_{2} \mathrm{~S}\left({ }^{79} \mathrm{Br}\right) 325.8435$.

\subsection{1,1'-(3,4-dibromothiophene-2,5-diyl)bis(propan-1-one) (7b)}

Prepared from diol $\mathbf{6 b}(45 \mathrm{mg}, 0.12 \mathrm{mmol})$ to obtain compound $\mathbf{8 b}$ as a colorless solid $(35 \mathrm{mg}$, $0.10 \mathrm{mmol}, 82 \%)$ Rf 0.70 (17:3 light petroleum:EtOAc); m.p. $118-120{ }^{\circ} \mathrm{C} ;{ }^{1} \mathrm{H}$ NMR $(400 \mathrm{MHz}$, $\left.\mathrm{CDCl}_{3}\right) \delta_{\mathrm{H}} 3.10\left(\mathrm{q}, J=7.1,4 \mathrm{H}, 2 \times \mathrm{CH}_{2}\right), 1.24\left(\mathrm{t}, J=7.1,6 \mathrm{H}, 2 \times \mathrm{CH}_{3}\right){ }^{13} \mathrm{C} N \mathrm{NMR}(100 \mathrm{MHz}$, $\left.\mathrm{CDCl}_{3}\right) \delta_{\mathrm{C}} 192.8,141.9,118.9,35.5,8.0$; IR(ATR) $\left(\mathrm{cm}^{-1}\right) v_{\max } 2983,2932,2878,1651,1451,1401$, 1379, 1348, 1275, 1184, 1083, 923, 890, 869; MS (ESI): MS (ESI): found 351.8751, calcd. for $\mathrm{C}_{10} \mathrm{H}_{10} \mathrm{Br}_{2} \mathrm{O}_{2} \mathrm{~S}\left({ }^{79} \mathrm{Br}\right) 351.8762$.

\subsection{1,1'-(3,4-dibromothiophene-2,5-diyl)bis(3-methylbutan-1-one) (7c)}

Prepared from diol $\mathbf{6 c}(0.50 \mathrm{~g}, 1.20 \mathrm{mmol})$ to obtain compound $\mathbf{7 c}$ as a colourless solid $(0.44 \mathrm{~g}, 90 \%)$; Rf 0.90 (8.5:1.5 light petroleum:EtOAc); m.p. $97-99{ }^{\circ} \mathrm{C} ;{ }^{1} \mathrm{H}$ NMR $\left(400 \mathrm{MHz}, \mathrm{CDCl}_{3}\right) \delta_{\mathrm{H}} 2.92$ $\left(\mathrm{d}, J=6.7 \mathrm{~Hz}, 4 \mathrm{H}, 2 \times \mathrm{CH}_{2}\right), 2.30(\mathrm{~m}, 2 \mathrm{H}, 2 \times \mathrm{CH}), 1.01\left(\mathrm{~d}, J=6.7 \mathrm{~Hz}, 12 \mathrm{H}, 4 \times \mathrm{CH}_{3}\right) ;{ }^{13} \mathrm{C} \mathrm{NMR}$ $\left(400 \mathrm{MHz}, \mathrm{CDCl}_{3}\right) \delta_{\mathrm{C}} 192.0,141.9,119.0,50.5,25.2,22.7$; IR(ATR) $\left(\mathrm{cm}^{-1}\right) v_{\max } 2956,2930,2871$, 1649, 1470, 1423, 1393, 1362, 1183, 1098, 1120, 1098, 984, 805, 767; MS (ESI): found 407.9384, calcd. for $\mathrm{C}_{14} \mathrm{H}_{18} \mathrm{Br}_{2} \mathrm{O}_{2} \mathrm{~S}\left({ }^{79} \mathrm{Br}\right) 407.9388$.

\subsection{1,1'-(3,4-dibromothiophene-2,5-diyl)bis(hexan-1-one) (7d)}

Prepared from diol $\mathbf{6 d}(0.52 \mathrm{~g}, 1.18 \mathrm{mmol})$ to obtain compound $\mathbf{7 c}$ as a colourless solid $(0.45 \mathrm{~g}, 87 \%)$; m.p. $\quad 71-72{ }^{\circ} \mathrm{C} ; \quad \mathrm{R} f 0.87$ (8:1 light petroleum:EtOAc); ${ }^{1} \mathrm{H} \quad \mathrm{NMR}\left(400 \mathrm{MHz}, \mathrm{CDCl}_{3}\right) \delta_{\mathrm{H}} 3.05$ $\left(\mathrm{t}, J=7.3 \mathrm{~Hz}, 4 \mathrm{H}, 2 \times \mathrm{CH}_{2}\right), 1.75\left(\mathrm{~m}, 4 \mathrm{H}, 2 \times \mathrm{CH}_{2}\right), 1.37\left(\mathrm{~m}, 8 \mathrm{H}, 2 \times \mathrm{CH}_{2} \mathrm{CH}_{2}\right), 0.91(\mathrm{t}, J=7.3 \mathrm{~Hz}, 6 \mathrm{H}$, $\left.2 \times \mathrm{CH}_{3}\right) ;{ }^{13} \mathrm{C}$ NMR $\left(100 \mathrm{MHz}, \mathrm{CDCl}_{3}\right) \delta_{\mathrm{C}} 192.5,141.9,118.9,41.9,31.4,23.8,22.6,14.0$. IR(ATR) $\left(\mathrm{cm}^{-1}\right) v_{\max } 2950,2918,2867,1666,1461,1426,1396,1370,1246,1173,952,870,848,72 ;$ MS (ESI): found 435.9707, calcd. for $\mathrm{C}_{16} \mathrm{H}_{22} \mathrm{Br}_{2} \mathrm{O}_{2} \mathrm{~S}\left({ }^{79} \mathrm{Br}\right) 435.9700$. 
4.12 1,1'-(3,4-dibromothiophene-2,5-diyl)bis(octan-1-one) (7e)

Prepared from diol $\mathbf{6 e}(0.29 \mathrm{~g}, 0.58 \mathrm{mmol})$ to obtain compound $7 \mathbf{e}$ as a faint yellow solid $(0.23 \mathrm{~g}$, $0.465 \mathrm{mmol}, 80 \%)$. Rf 0.74 (9:1 light petroleum:EtOAc); m.p. $48-50{ }^{\circ} \mathrm{C} ;{ }^{1} \mathrm{H}$ NMR $(500 \mathrm{MHz}$, $\left.\mathrm{CDCl}_{3}\right) \delta_{\mathrm{H}} 3.05\left(\mathrm{t}, J=6.7 \mathrm{~Hz}, 4 \mathrm{H}, 2 \times \mathrm{CH}_{2}\right), 1.76-1.71\left(\mathrm{~m}, 4 \mathrm{H}, 2 \times \mathrm{CH}_{2}\right), 1.38-1.25(\mathrm{~m}, 16 \mathrm{H}$, $\left.2 \times \mathrm{CH}_{2} \mathrm{CH}_{2} \mathrm{CH}_{2} \mathrm{CH}_{2} \mathrm{CH}_{2}\right), 0.88\left(\mathrm{t}, J=6.7 \mathrm{~Hz}, 6 \mathrm{H}, 2 \times \mathrm{CH}_{3}\right) ;{ }^{13} \mathrm{C}$ NMR $\left(125 \mathrm{MHz}, \mathrm{CDCl}_{3}\right) \delta_{\mathrm{C}} 192.6$, $141.9,119.0,41.9,31.8,29.2,29.2,24.2,22.7,14.2$; IR(ATR) $\left(\mathrm{cm}^{-1}\right) v_{\max } 2954,2916,2847,1666,1559$, 1470, 1269, 1222, 1171, 770, 727; MS (ESI): found 492.0300, calcd. for $\mathrm{C}_{20} \mathrm{H}_{30} \mathrm{Br}_{2} \mathrm{O}_{2} \mathrm{~S}\left({ }^{79} \mathrm{Br}\right) 492.0300$.

\subsection{1,1'-(3,4-(ethylenedithio)thiophene-2,5-diyl)bis(3-methylbutan-1-one) (8a)}

Neat 1,2-ethanedithiol $(0.84 \mathrm{~g}, 9.01 \mathrm{mmol})$ was added slowly to a solution of $(7 \mathrm{c})(1.68 \mathrm{~g}, 4.10 \mathrm{mmol})$ and $\mathrm{Na}_{2} \mathrm{CO}_{3}(1.00 \mathrm{~g}, 9.44 \mathrm{mmol})$ in dry DMF $(20.5 \mathrm{~mL})$ and the mixture was stirred at room temperature under an atmosphere of argon. After $(64 \mathrm{~h})$ the mixture was extracted with ethyl acetate $(3 \times 100 \mathrm{~mL})$. The organic layer was washed with $2 \mathrm{M} \mathrm{HCl}(3 \times 30 \mathrm{~mL})$ and then dried with $\mathrm{MgSO}_{4}$, filtered and concentrated under reduced pressure. Purification was achieved by flash column chromatography (18:1 light petroleum:EtOAc) to afford $\mathbf{8 a}(0.991 \mathrm{~g}, 2.89 \mathrm{mmol}, 70 \%)$ as yellow microcrystals; Rf0.26 (18:1 light petroleum:EtOAc); m.p. $99-100{ }^{\circ} \mathrm{C} ;{ }^{1} \mathrm{H}$ NMR $(400 \mathrm{MHz}$, $\left.\mathrm{CDCl}_{3}\right) \delta_{\mathrm{H}} 3.21\left(\mathrm{~s}, 4 \mathrm{H}, 2 \times \mathrm{CH}_{2}\right), 2.69\left(\mathrm{~d}, J=6.7 \mathrm{~Hz}, 4 \mathrm{H}, 2 \times \mathrm{CH}_{2} \mathrm{CO}\right), 2.36-2.28(\mathrm{~m}, 2 \mathrm{H}, 2 \times \mathrm{CH})$, $1.00\left(\mathrm{~d}, J=6.7 \mathrm{~Hz}, 12 \mathrm{H}, 4 \times \mathrm{CH}_{3}\right) ;{ }^{13} \mathrm{C}$ NMR $\left(100 \mathrm{MHz}, \mathrm{CDCl}_{3}\right) \delta_{\mathrm{C}} 192.9,136.4,133.5,50.1,26.5$, 25.7, 22.8; IR(ATR) $\left(\mathrm{cm}^{-1}\right) v_{\max } 2951,2871,1728,1654,1442,1411,1398,1362,1278,1183,1035$, 956, 917, 880, 688; MS (EI): found 342.0785, calcd. for $\mathrm{C}_{16} \mathrm{H}_{22} \mathrm{O}_{2} \mathrm{~S}_{3} 342.0782$. Anal. Calcd. for $\mathrm{C}_{16} \mathrm{H}_{22} \mathrm{O}_{2} \mathrm{~S}_{3} \mathrm{C}: 56.10 ; \mathrm{H}, 6.47 \%$ found C: 55.97, $\mathrm{H}: 6.78 \%$.

\subsection{1,1'-(3,4-(ethylenedithio)thiophene-2,5-diyl) bis(hexan-1-one) (8b)}

Neat 1,2-ethanedithiol $(83.0 \mathrm{mg}, 0.89 \mathrm{mmol})$ was added to a solution of $7 \mathbf{d}(156 \mathrm{mg}, 0.36 \mathrm{mmol})$ and $\mathrm{Na}_{2} \mathrm{CO}_{3}(94 \mathrm{mg}, 0.89 \mathrm{mmol})$ in dry DMF $(1.8 \mathrm{~mL})$ and the mixture was stired at room temperature under an atmosphere of argon $(40 \mathrm{~h})$. The mixture was extracted with ethyl acetate $(3 \times 4 \mathrm{~mL})$ and the organic layer was then washed with $(2 \mathrm{M} \mathrm{HCl})$ until DMF free. The organic extracts were dried with $\mathrm{MgSO}_{4}$ and concentrated in vacuo. The crude product was then purified by flash column chromatography (9:1 light petroleum:EtOAc) giving a pale yellow solid (75.0 $\mathrm{mg}, 57 \%)$; $\mathrm{R} f 0.47$ (9:1 light petroleum:EtOAc); m.p. 59-60 ${ }^{\circ} \mathrm{C} ;{ }^{1} \mathrm{H}$ NMR $\left(400 \mathrm{MHz}, \mathrm{CDCl}_{3}\right) \delta_{\mathrm{H}} 3.22\left(\mathrm{~s}, 4 \mathrm{H}, 2 \times \mathrm{CH}_{2}\right), 2.28$ $\left(\mathrm{t}, J=7.2 \mathrm{~Hz}, 4 \mathrm{H}, 2 \times \mathrm{CH}_{2}\right), 1.74-1.70\left(\mathrm{~m}, 4 \mathrm{H}, 2 \times \mathrm{CH}_{2}\right), 1.34-1.31\left(\mathrm{~m}, 8 \mathrm{H}, 2 \times \mathrm{CH}_{2} \mathrm{CH}_{2}\right), 0.91$ $\left(\mathrm{t}, J=7.2,6 \mathrm{H}, 2 \times \mathrm{CH}_{3}\right) ;{ }^{13} \mathrm{C} \mathrm{NMR}\left(100 \mathrm{MHz}, \mathrm{CDCl}_{3}\right) \delta_{\mathrm{C}} 193.3,136.4,133.2,41.2,31.5,26.5,24.3$, 22.6, 14.1; IR(ATR) $\left(\mathrm{cm}^{-1}\right) v_{\max } 2954,2933,2859,1641,1463,1447,1417,1366,1315,1275,1247$, 1184, 1164, 973, 848, 730; MS (EI): $m / 2$ found 370.1095, calcd. for $\mathrm{C}_{18} \mathrm{H}_{26} \mathrm{O}_{2} \mathrm{~S}_{3} 370.1086$.

\subsection{1,1'-(3,4-(ethylenedithio)thiophene-2,5-diyl)bis(octan-1-one) (8c)}

Neat 1,2-ethanedithiol $(83 \mathrm{mg}, 0.9 \mathrm{mmol})$ was added to a solution of $7 \mathbf{e}(194 \mathrm{mg}, 0.39 \mathrm{mmol})$ and $\mathrm{Na}_{2} \mathrm{CO}_{3}(104 \mathrm{mg}, 0.98 \mathrm{mmol})$ in dry DMF $(4 \mathrm{~mL})$ at room temperature. The mixture was then stirred for $(40 \mathrm{~h})$ until a precipitate formed. The mixture was extracted with ethyl acetate $(4 \times 10 \mathrm{~mL})$ and the organic layer was then washed with $(2 \mathrm{M} \mathrm{HCl})$ until DMF free. The extracts were dried $\left(\mathrm{MgSO}_{4}\right)$ and concentrated in vасио. The crude product was purified by flash column chromatography (9:1 light petroleum:EtOAc) giving a butter cream yellow solid $(75 \mathrm{mg}, 45 \%) ; \mathrm{R} f 0.26$ (9:1 light petroleum:EtOAc); m.p. $65-67{ }^{\circ} \mathrm{C} ;{ }^{1} \mathrm{H}$ NMR $\left(400 \mathrm{MHz}, \mathrm{CDCl}_{3}\right) \delta_{\mathrm{H}} 3.20\left(\mathrm{~s}, 4 \mathrm{H}, 2 \times \mathrm{CH}_{2}\right), 2.20$ $\left(\mathrm{t}, J=7.4 \mathrm{~Hz}, \quad 4 \mathrm{H}, \quad 2 \times \mathrm{CH}_{2}\right), \quad 1.76-1.69 \quad\left(\mathrm{~m}, \quad 4 \mathrm{H}, \quad 2 \times \mathrm{CH}_{2}\right), \quad 1.34-1.29 \quad(\mathrm{~m}, \quad 16 \mathrm{H}$, $\left.2 \times \mathrm{CH}_{2} \mathrm{CH}_{2} \mathrm{CH}_{2} \mathrm{CH}_{2} \mathrm{CH}_{2}\right), 0.86\left(\mathrm{t}, J=7.4,6 \mathrm{H}, 2 \times \mathrm{CH}_{3}\right) ;{ }^{13} \mathrm{C} \mathrm{NMR}\left(100 \mathrm{MHz}, \mathrm{CDCl}_{3}\right) \delta_{\mathrm{C}} 193.2,136.3$, 133.1, 41.2, 31.8, 29.3, 29.1, 26.5, 24.5, 22.7, 14.2; IR (ATR) $\left(\mathrm{cm}^{-1}\right) v_{\max } 2953,2928,2850,1646,1465$, 1444, 1418, 1404, 1368, 1354, 1316, 1298, 1250, 1181, 1166, 1099, 1057, 1021, 970; MS (EI): found 426.1721, calcd. for $\mathrm{C}_{22} \mathrm{H}_{34} \mathrm{O}_{2} \mathrm{~S}_{3} 426.1721$.

\subsection{2,2' -Thiodiacetonitrile ( 12 )}

Solid $\mathrm{Na}_{2} \mathrm{~S} \cdot 9 \mathrm{H}_{2} \mathrm{O}(10.8 \mathrm{~g}, 45.01 \mathrm{mmol})$ was dissolved in DME $(35 \mathrm{~mL})$, and chloroacetonitrile $(4.77 \mathrm{~g}$, $63.2 \mathrm{mmol})$ was then added. The mixture was allowed to stir at r.t. $(16 \mathrm{~h})$. Water was added and the compound was extracted from chloroform $(3 \times 40 \mathrm{~mL})$. The organic layer was dried $\left(\mathrm{MgSO}_{4}\right)$ and the 
solvent removed. Elution through a silica plug (1:1 EtOAc:pentane) yielded compound $\mathbf{1 6}$ as a colorless oil $(3.24 \mathrm{~g}, 28.9 \mathrm{mmol}, 91 \%) ;{ }^{1} \mathrm{H}$ NMR $\left(400 \mathrm{MHz}, \mathrm{CDCl}_{3}\right) \delta_{\mathrm{H}} 3.57(\mathrm{~s}, 4 \mathrm{H}) ;{ }^{13} \mathrm{C} \mathrm{NMR}(100 \mathrm{MHz}$, $\left.\mathrm{CDCl}_{3}\right) \delta_{\mathrm{C}} 114.9,17.5$; IR(ATR) $\left(\mathrm{cm}^{-1}\right) v_{\max } 2970,2929,2246,1399,1174,922$; MS (EI): found 112.0089, calcd. for $\mathrm{C}_{4} \mathrm{H}_{4} \mathrm{~N}_{2} \mathrm{~S}$ requires 112.0089. The above values are concordant with literature data. $\underline{23}$

\subsection{Potassium 2,5-dicyanothiophene-3,4-bis(olate) (13)}

To dry $t \mathrm{BuOK}(8.0 \mathrm{~g}, 71.3 \mathrm{mmol})$ in dry THF $(95 \mathrm{~mL})$, a solution of $\mathbf{1 2}(4.00 \mathrm{~g}, 35.6 \mathrm{mmol})$ in THF $(43 \mathrm{~mL})$ was added dropwise at $0{ }^{\circ} \mathrm{C}$. The mixture was stirred for $5 \mathrm{~min}$ and then added a solution of diethyl oxalate $(5.21 \mathrm{~g}, 35.66 \mathrm{mmol})$ in dry THF $(40 \mathrm{~mL})$ at $0{ }^{\circ} \mathrm{C}$. After $30 \mathrm{~min}$ stirring at $0{ }^{\circ} \mathrm{C}$ the precipitate was collected by vacuum filtration and washed with diethyl ether and THF to obtain $\mathbf{1 3}$ as a brown powder $(7.26 \mathrm{~g}, 84 \%) ;{ }^{13} \mathrm{C}$ NMR $\left(100 \mathrm{MHz}, \mathrm{DMSO}-d_{6}\right) \delta_{\mathrm{C}} 174.9,122.8,65.7$; IR (ATR) $\left(\mathrm{cm}^{-1}\right) v_{\max } 2168,1712,1518,1315,1229,1019,764,592$. The compound was used as obtained directly.

\subsection{3,4-Ethylenedioxythiophene-2,5-dicarbonitrile (14)}

Under an argon atmosphere potassium salt $\mathbf{1 3}(0.50 \mathrm{~g}, 2.06 \mathrm{mmol})$ was dissolved in anhydrous DMF $(1.5 \mathrm{~mL})$ at room temperature, and dry potassium carbonate $(0.11 \mathrm{~g}, 0.83 \mathrm{mmol})$ added followed by tetrabutylammonium bromide $(35 \mathrm{mg}, 0.10 \mathrm{mmol})$. The mixture was heated to $135{ }^{\circ} \mathrm{C}$ and $1,2-$ dichloroethane $(0.41 \mathrm{~g}, 4.13 \mathrm{mmol})$ added dropwise. After stirring at $135{ }^{\circ} \mathrm{C}(5 \mathrm{~h})$ the reaction was stopped and allowed to cool down. The mixture was extracted with ethyl acetate $(3 \times 10 \mathrm{~mL})$, washed with $5 \% \mathrm{w} / \mathrm{w} \mathrm{LiCl}(\mathrm{aq})$ solution $(3 \times 10 \mathrm{~mL})$ and the combined organic extracts dried $\left(\mathrm{MgSO}_{4}\right)$ and concentrated in vacuo to afford the crude product. Filtering through a silica plug with a dichloroethane wash yielded, on removal of the solvent, $14(0.13 \mathrm{~g}, 35 \%)$ obtained as a colourless solid, R $f 0.68$ (1:1 EtOAc:hexane) ${ }^{1} \mathrm{H} \quad \mathrm{NMR} \quad\left(400 \mathrm{MHz}, \mathrm{CDCl}_{3}\right): \delta_{\mathrm{H}} 4.42\left(\mathrm{~s}, \quad 4 \mathrm{H}, 2 \times \mathrm{CH}_{2}\right),{ }^{13} \mathrm{C} \quad \mathrm{NMR} \quad(100 \mathrm{MHz}$, $\left.\mathrm{CDCl}_{3}\right): \delta_{\mathrm{C}} 147.8,110.6,91.3,65.2$. IR (ATR) $\left(\mathrm{cm}^{-1}\right) v_{\max } 2946,2215,1632,1504,1449,1078,914$, $843,806 \mathrm{~cm}^{-1}$. MS (EI): found 191.9988, calcd. for $\mathrm{C}_{8} \mathrm{H}_{4} \mathrm{~N}_{2} \mathrm{O}_{2} \mathrm{~S} 191.9993$.

\subsection{3,4-Ethylenedioxythiophene-2,5- dicarbaldehyde (15)}

A solution of DIBAL-H (1.08 mL, $1.2 \mathrm{M} 20 \%$ wt. in toluene) was added dropwise at $0{ }^{\circ} \mathrm{C}$ to a solution of $14(100 \mathrm{mg}, 0.52 \mathrm{mmol})$ in dry toluene $(5.2 \mathrm{~mL})$. The reaction was stirred $(30 \mathrm{~min})$ at $0{ }^{\circ} \mathrm{C}$ until the starting material was consumed as judged by TLC analysis. The reaction mixture was quenched with $(1 \mathrm{M}, \mathrm{HCl})$ and extracted with ethyl acetate $(3 \times 10 \mathrm{~mL})$ and dried $\left(\mathrm{MgSO}_{4}\right)$, the solvent was evaporated to yield the desired dialdehyde $\mathbf{1 5}(83 \mathrm{mg}, 80 \%)$ as a tan brown solid. Rf 0.64 (EtOAc); ${ }^{1} \mathrm{H}$ NMR $\left(400 \mathrm{MHz}, \mathrm{CDCl}_{3}\right) \delta_{\mathrm{H}} 10.04(\mathrm{~s}, 2 \mathrm{H}, 2 \times \mathrm{CHO}), 4.45\left(\mathrm{~s}, \quad 4 \mathrm{H}, 2 \times \mathrm{CH}_{2}\right) ;{ }^{13} \mathrm{C} \quad \mathrm{NMR}(100 \mathrm{MHz}$, $\left.\mathrm{CDCl}_{3}\right) \delta_{\mathrm{C}} 181.0,147.4,124.2,65.0$; IR(ATR) $\left(\mathrm{cm}^{-1}\right) v_{\max }$ 2953, 2922, 2851, 1662, 1643, 1260, 1230; MS (EI): found 197.9987, calcd. for $\mathrm{C}_{8} \mathrm{H}_{6} \mathrm{O}_{4} \mathrm{~S}$ 197.9987. These values are consistent with published literature. ${ }^{11 \mathrm{~d}}$

\section{Acknowledgements}

One of us (MA) would like to thank The Higher Committee for Education Development in Iraq (HCEDIraq) for the providing of a scholarship. The University of Nottingham is acknowledged for additional support.

\section{Appendix A. Supplementary data}

Supplementary data related to this article can be found at http://dx.doi.org/10.1016/j.tet.2017.08.026.

\section{References}

1. For comprehensive reviews of the general area of thiophene materials from early developments until 2009 see:(a) R.D. McCullough, Adv Mater. 10, 1998, 93-116;

(b) A. Mishra, C.-Q. Ma and P. Bäuerle, Chem Rev 109, 2009, 1141-1276;

(c) I.F. Perepichka and D.F. Perepichka, (Eds.), Handbook of Thiophene-based Materials: Applications in Organic Electronics and Photonics 1-2, 1, 2009, John Wiley \& Sons, 1-910.

2. Overviews of recent, 2009-, (oligo)thiophene developments:(a) L. Zhang, N.S. Colella, B.P. Cherniawski, S.C.B. Mannsfeld and A.L. Briseno, ACS Appl Mater. Interfaces 6, 2014, 5327-5343;

(b) A.L. Kanibolotsky, N.J. Findlay and P.J. Skabara, Beilstein J Org Chem 11, 2015, 1749-1766; 
(c) M. Iyoda and H. Shimizua, Chem Soc Rev 44, 2015, 6411-6424, and references therein.

3. (a) E. Bundgaard and F.C. Krebs, Sol Energy Mater Sol Cells 91, 2007, 954-985;

(b) G. Li, R. Zhu and Y. Yang, Nat Photonics 6, 2012, 153-161;

(c) V. Malytskyi, J.-J. Simon, L. Patrone and J.-M. Raimundo, RSC Adv 5, 2015, 354-397.

4. (a) C.B. Nielsen and I. McCulloch, Prog Poly Sci 38, 2013, 2053-2069;

(b) Y. Olivier, D. Niedzialek, V. Lemaur, et al., Adv Mater. 26, 2014, 2119-2136.

5. T.-P. Huynh, P.S. Sharma, M. Sosnowska, F. D'Souza and W. Kutner, Prog Poly Sci 47, 2015, 1-25.

6. (a) P.M. Beaujuge and J.R. Reynolds, Chem Rev 110, 2010, 268-320;

(b) P.M. Beaujuge, C.M. Amb and J.R. Reynolds, Acc Chem Res 43, 2010, 1396-1407;

(c) A.C. Grimsdale, K.L. Chan, R.L. Martin, P.G. Jokisz and A.B. Holmes, Chem Rev 109, 2009, $897-$ 1091.

7. Overviews of PDOT:(a) L.B. Groenendaal, F.H. Jonas, D. Freitag, H. Pielartzik and J.R. Reynolds, Adv Mater 12, 2000, 481-494;

(b) J. Roncali, P. Blanchard and P. Frère, J Mater. Chem 15, 2005, 1589-1610;

(c) S. Kirchmeyer and K. Reuter, J Mater. Chem 15, 2005, 2077-2088.

8. For overviews of this area see:(a) P.J. Taroni, I. Hoces, N. Stingelin, M. Heeney and E. Bilotti, Isrl J Chem 54, 2014, 534-552;

(b) L.M. Cowen, J. Atoyo, M.J. Carnie, D. Baran and B.C. Schroeder, ECS J Solid State Sci Technol 6, 2017, N3080-N3088 and citations thereof.

9. For example, electrical conductivities $>4000 \mathrm{~S} \mathrm{~cm}-1$ are knownN. Kim, S. Kee, S.H. Lee, et al., Adv Mater 26, 2014, 2109.

10. (a) C. Wang, J.L. Schindler, C.R. Kannewurf and M.G. Kanatzidis, Chem Mater 7, 1995, 58-68;

(b) M. Turbiez, P. Frère, M. Allain, N. Gallego-Planas and J. Roncali, Macromolecules 38, 2005, 68066812.

11. For heteroatom and vinyligous modifications see for example:(a) E. Poverenov, Y. Sheynin, N. Zamoshchik, et al., J Mater. Chem 22, 2012, 14645-14655;

(b) N.F. Montcada, R. Domínguez, B. Pelado, P. De La Cruz, E. Palomares and F. Langa, J Mat Chem A 3, 2015, 11340-11348;

(c) P.M. Burrezo, B. Pelado, R.P. Ortiz, et al., Chem Eur J 21, 2015, 1713-1725;

(d) S. Akoudad, P. Frere, N. Mercier and J. Roncali, J Org Chem 64, 1999, 4267-4272.

12. M. Schnurch, M. Spina, A.F. Khan, M.D. Mihovilovic and P. Stanetty, Chem Soc Rev 36, 2007, 1046-1057.

13. H. Pang, P.J. Skabara, S. Gordeyev, J. McDouall, S.J. Coles and M.B. Hursthouse, Chem Mater. 19, 2007, 301-307.

14. (a) S. Benz, M. Macchione, Q. Verolet, J. Mareda, N. Sakai Naomi and S. Matile, J Am Chem Soc 138, 2016, 9093-9096;

(b) M. He and F. Zhang, J Org Chem 72, 2007, 442-451.

15. (a) H. Lin, W. Xu and D. Zhu, J Mat Chem 20, 2010, 884-890;

(b) D.T. Tùng, D.T. Tuân, N. Rasool, et al., Adv Synth Catal 351, 2009, 1595-1609.

16. S. Chen, B. Lu, X. Duan and J. Xu, J Poly Sci 50, 2012, 1967-1978.

17. (a) P. Blanchard, B. Jousselme, P. Frère and J. Roncali, J Org Chem 67, 2002, 3961-3964;

(b) P. Blanchard, A. Cappon, E. Levillain, Y. Nicolas, P. Frère and J. Roncali, Org Lett 4, 2002, 607 609.

18. L. Groenendaal, G. Zotti, P.-H. Aubert, S.M. Waybright and J.R. Reynolds, Adv Mater 15, 2003, $855-879$. 
19. A.K. Mohanakrishnan, A. Hucke, M.A. Lyon, M.V. Lakshmikantham and M.P. Cava, Tetrahedron 55, 1999, 11745-11754.

20. Present attempts to prepare the 3,4-ethylenedithio analogue of 15 (i.e. $8, \mathrm{R}=\mathrm{H}$ ) are complicated by dithioacetal formation and other competing reactions

21. P.L. Fuchs, A.B. Charette, T. Rovis and J.W. Bode, Essential Reagents for Organic Synthesis, 2016, Wiley; New York, . ISBN: 978-1-119-27830-6250.

22. (a) K. Araki, H. Endo, G. Masuda and T. Ogawa, Chem Eur J 10, 2004, 3331-3340;

(b) A. Honciuc, R.M. Metzger, A. Gong and C.W. Spangler, J Am Chem Soc 129, 2007, 8310-8319.

23. P. Kielbasinski, M. Rachwalski, M. Mikolajczyk, et al., Adv Synth Catal 349, 2007, 1387-1392. 